\title{
SYZYGIES OF MODULES AND APPLICATIONS TO PROPAGATION OF REGULARITY PHENOMENA
}

\author{
A. Meril and D.C. Struppa
}

\begin{abstract}
Propagation of regularity is considered for solutions of rectangular sys. tems of infinite order partial differential equations (resp. convolution equations) in spaces of hyperfunctions (resp. $C^{\infty}$ functions and distributions). Known results of this kind are recovered as particular cases, when finite order partial differential equations are considered.
\end{abstract}

\section{Introduction}

In [10], exploiting some older ideas of Bochner [5], Ehrenpreis was the first to explicitly consider the famous Hartogs' theorem on the removability of compact singularities, in the framework of overdetermined systems of linear partial differential equations. His ideas were subsequently exploited by various authors in several papers, among which we may recall [9], [14], [15], [20], [23], [25], (this list, however, is not at all exhaustive). The general problem which is addressed in these papers can be roughly described as follows:

Problem. Let $\Omega$ be an open subset of $\mathbb{R}^{n}$, and let $K \subset \Omega$ be a compact subset. Let $f$ be a (generalized) function defined on $\Omega \backslash K$, which is a solution of an overdetermined system of equations

$$
T_{1}(f)=\cdots=T_{q}(f)=0
$$

with $T_{1}, \ldots, T_{q}$ suitable linear continuous operators. We seek conditions on $T_{1}, \ldots, T_{q}$ so that $f$ extends to a (generalized) function $f$, defined on $\Omega$, and such that

$$
T_{1}(\tilde{f})=\cdots=T_{q}(\tilde{f})=0 .
$$

In the papers we quoted before, as well as in other ones, this problem is studied when $f$ is a $C^{\infty}$ function, a distribution, an ultradistribution or a hyperfunction, while the operators $T_{i}$ are linear partial differential operators (both with constant and with variable coefficients), convolution operators, infinite 
order differential operators (see, however, $[27]$ for a fairly complete historical survey of this problem).

With the exception of [9], [14], [20], [25], though, nobody has ever attempted to deal with the case in which (1.1) is replaced by a $q \times s$ system of equations and, in the few cases in which this is done, the known results are quite limited (only constant coefficients partial differential equations are treated) and unsatisfactory, since either very heavy hypotheses are requested, and no concrete examples are given, or only the Cauchy-Riemann system is treated.

In this paper, therefore, we deal with the task of providing a complete satisfactory treatment for such a phenomenon, in a large class of spaces and of operators.

It turns out that the problem we study has two main components: the first (which does not appear in the simple case of equation (1.1)) is totally algebraic in nature and relates to the construction of the first syzygies of modules on suitable rings of analytic functions; this problem is discussed in section 2, and our first result (Theorem 2.1) gives a very explicit condition on a module which implies a simple concrete representation of it syzygies; we think this result is of some independent interest. The complete description of the syzygies, however, is not necessary for the understanding of our propagation of regularity phenomenon, and therefore we use the important work [1] of Andreotti and Nacinovich to provide a simple condition which will suffice for our subsequent results. In particular we show that the hypotheses which we need to ask are a perfectly natural generalization of Ehrenpreis' ones [10]. The second important component is analytic in nature, and relates to the possibility of providing theorems on division with bounds in several spaces of entire functions: we quote, in section 3 , a few known results, and we then proceed to prove new and important division theorems (Theorems 3.5 and 3.6) in the space $\hat{\mathcal{D}}$ of Fourier transforms of compactly supported differentiable functions: such results will probably be useful in many other problems in the theory of convolution equations in $\mathcal{D}^{\prime}$. We finally prove our main results in section 4 . Here, three propagation of regularity theorems are proved, in different, and equally interesting situations. Theorem 4.1 deals with (rectangular) systems of linear constant coefficients partial differential operators in spaces of $C^{\infty}$ functions and of distributions. Theorem 4.3 concerns systems of infinite order differential equations for hyperfunctions on $\mathbb{R}^{n}$, while Theorem 4.4 (technically, the most complex) generalizes Theorem 4.1 to the case in which convolution operators are considered. Several explicit examples are finally considered.

Acknowledgements. The first author wishes to thank the French Ministry of General Education for granting him a leave of absence, and the Dept. of Mathematics of the University of Maryland, for the kind hospitality during the period in which this paper was written. The second author thanks the Dept. of Mathematics of the University of Calabria, for aliowing him to take a leave of absence, and the Dept. of Math. Sciences of George Mason University for the kind hospitality. The second author also acknowledges the Grant from M.P.I. of 
the Italian Government for researches in complex analysis, which has partially supported this work. We both finally wish to thank Ahmed Sebbar for many useful suggestions, as well as the referee for his constructive criticism.

\section{Algebraic Techniques}

Throughout this paper $R$ will be a commutative unitary noetherian ring and $M$ will be a finitely generated $R$-module with finite basis $\left\{e_{1}, \ldots, e_{q}\right\}$. As it is well known (see e.g. [28]), the submodule $S(M)$ of $R^{q}$ of the elements $\left\{a_{1}, \ldots, a_{q}\right\} \in R^{q}$ such that

$$
a_{1} e_{1}+\cdots+a_{q} c_{q}=0
$$

is called the module of relations of $\left\{e_{1}, \ldots, e_{q}\right\}$. Such a module is often also called the first module of syzygies of $M$, with respect to the basis $\left\{e_{1}, \ldots, e_{q}\right\}$. We recall, $[7\}$, that two $R$-modules $M_{1}, M_{2}$ are said to be equivalent if there exist two free $R$-modules $N_{1}, N_{2}$ such that $M_{1} \oplus N_{1}$ and $M_{2} \oplus N_{2}$ are isomorphic. One then sees that $S(M)$ depends on the choice of the basis, but that two first modules of syzygies of an $R$-module $M$ with respect to different bases are equivalent. (see [28, Lemma 1 p. 238]).

This notion was first developed by Hilbert [13] in connection with basic problems in algebraic geometry.

In the general case, the explicit construction of the generators of $S(M)$ is quite complicated, and it often relies on the use of the so called Koszul complex $[22]$ or of the generalized Koszul complex [1], [6], [7], [8], [16].

Let us briefly discuss these two objects, as they will give us some insight on the computation of syzygies. Take, with the notation introduced before, $e_{1}, \ldots, e_{q}$ in $R$, and consider the indeterminates $t_{1}, \ldots, t_{q}$. We introduce the exterior $R$-algebra $K$ on $t_{1}, \ldots, t_{q}$ so that $K$ is a graded algebra with components $K_{0}=R, K_{1}, \ldots, K_{q}$ and each $K,(0 \leq j \leq m)$ has an $R$-base consisting of all possible products

$$
t_{i_{1}} \wedge t_{i_{2}} \wedge \cdots \wedge t_{i_{j}}\left(1 \leq i_{1}<\cdots<i_{j} \leq q\right) .
$$

$K$ can then be turned into a complex (the Koszul complex) by defining the following maps:

$$
0 \longrightarrow K_{q} \stackrel{d_{q}}{\longrightarrow} K_{q-1} \longrightarrow \cdots \longrightarrow K_{1} \stackrel{d_{i}}{\longrightarrow} K_{0} \longrightarrow 0
$$

where

$$
d_{j}\left(t_{i_{2}} \wedge \cdots \wedge t_{i_{j}}\right)=\sum_{p=1}^{j}(-1)^{p+1} e_{i_{p}} t_{i_{1}} \wedge \cdots \wedge \hat{t}_{i_{p}} \wedge \cdots \wedge t_{i_{j}}
$$


where the symbol ' means that the specific term has to be omitted. Note that while $K_{0}=R$, one has $K_{1}=R^{g}$ and

$$
d_{1}: K_{1} \longrightarrow K_{0}
$$

acts as follows:

$$
d_{1}\left(a_{1} t_{1}+\cdots+a_{q} t_{q}\right)=a_{1} e_{1}+\cdots+a_{4} e_{q}
$$

one has therefore that ker $d_{1}=S(M)$, where $M$ is the $R$-module generated by $e_{1}, \ldots, e_{q}$. It is also easy to check that (2.2) makes $K$ into a complex, i.e. $d_{i+1} \circ d_{i}=0$ for any $i=1, \ldots, q-1$. Of course it is not true, in general, that this complex is exact, but if this wcre true (at least at the first step), then we would have a simple description of $S(M)$. In particular we note, for future reference, that an $R$-basis for $K_{2}$ is given by the monomials

$$
t_{i} \wedge t_{j} \quad 1 \leq i<j \leq q,
$$

and therefore $\operatorname{Im}\left(d_{2}\right)$ is generated by

$$
d_{2}\left(t_{i} \wedge t_{j}\right)=e_{i} t_{i}-e_{j} t_{j}
$$

The following definition is standard in commutative algebra, see e.g. [22]:

Definition 2.1. Let $P_{1}, \ldots, P_{q}$ be $q$ elements in the ring $R$. We say that $\left(P_{1}, \ldots, P_{q}\right)$ is a regular sequence if, for any $i=1, \ldots, q, P_{i}$ is not a zero divisor in the quotient ring

$$
R /\left(R \cdot P_{1}+\cdots+R \cdot P_{i-1}\right) .
$$

Note that the definition depends on the order in which we consider the elements $P_{1}, \ldots, P_{q}$; it is true however [22] that, if $R$ is a local ring, the notion of regular sequence does not depend on the order any longer.

The interest of this definition is that, if $P_{1}, \ldots, P_{q}$ is a regular sequence, them $\operatorname{Im}\left(d_{2}\right)=\operatorname{ker}\left(d_{1}\right)$, in the Koszul complex associated to the $q$-tuple $\left(P_{1}, \ldots, P_{q}\right)$ (see [21], [23], [24]); therefore, if $M=R \cdot P_{1}+\cdots+R \cdot P_{q}$ (i.e. if $M$ is the ideal generated by $\left.P_{1}, \ldots, P_{q}\right)$, and if $\left(P_{1}, \ldots, P_{q}\right)$ is a regular sequence, we have an explicit description of $S(M)$; it has however to be noted that, in general, the computation of $S(M)$ is an extremely difficult and complex procedure; in view of the great interest for an explicit solution to this problem (interest which stems from problems in algebraic geometry, systems control theory, inage reconstruction, etc.), many papers have becn devoted to this aspect, starting with the classical works of Hermann [12], Lazard [19], up to the more recent contributions of Bayer and Stillman [22]; we refer the interested reader to this last work for references.

If $R$ is a noetherian ring and $P_{1}, \ldots, P_{q}$ are such that $\left(P_{1}, \ldots, P_{q}\right)=R$, then the Koszul complex is exact. In particular, in the special case in which 
$R=k\left[x_{1}, \ldots, x_{n}\right]$ is a polynomial ring on a field $k$, then a family $\left(P_{3}, \ldots, P_{q}\right)$ of polynomials, such that $V=\left\{x \in k^{n}: P_{1}(x)=\cdots=P_{q}(x)=0\right\}=\phi$, while not always a regular sequence, does however satisfy the requirement that $\operatorname{Im}\left(d_{2}\right)=\operatorname{ker}\left(d_{1}\right),[21],[23] ;$ on the other hand, if $q=2$, then $\left(P_{1}, P_{2}\right)$ form a regular sequence if and only if they have no common zeroes: for $q \geq 3$ this is not true in general. Finally, the algebraic notion of regular sequence corresponds to the geometrical notion of complete intersection: $\left(P_{1}, \ldots, P_{q}\right)$ is a regular sequence if and only if, for every $j=1, \ldots, q$, the variety

$$
V_{j}=\left\{x \in k^{n}: P_{1}(x)=\cdots=P_{j}(x)=0\right\}
$$

has codimension $j$, or it is empty (see e.g. $[1\}$ ).

By using this property, we can easily construct examples of regular sequences in $\mathbb{C}\left[x_{1}, \ldots, x_{n}\right]$, such as

$$
\left(P_{1}, \ldots, P_{q}\right)=\left(x_{1}, \ldots, x_{q}\right)
$$

and, fo: $n=3$,

$$
\left(P_{1}, P_{2}, P_{3}\right)=(x(y-1), y, z(y-1)) .
$$

In this last example $\left(P_{1}, P_{2}, P_{3}\right)$ is a regular sequence, while $\left(P_{1}, P_{3}, P_{2}\right)$ is not.

We will say that a sequence is very regular if all of its permutations are regular.

Let us now turn to the generalized Koszul complex. In the same way in which the Koszul complex can be thought of as a complex associated with the map

$$
f=R^{q} \longrightarrow R
$$

defined by

$$
f\left(a_{1}, \ldots, a_{q}\right)=e_{1} a_{1}+\cdots+e_{q} a_{q},
$$

so we can define (see, actually $[1],[6],[7],[8],[16]$ ) the generalized Koszul complex associated to a map

$$
f: R^{q} \longrightarrow R^{s} \quad, \quad q \geq s
$$

this corresponds to replacing the vector $\left(e_{1}, \ldots, e_{q}\right)$ by a matrix $\left(e_{i j}\right), i=$ $1, \ldots, s, j=1, \ldots, q$. We will not get into the lengthy construction of such a complex, except for noting that it is essentially an iteration of the usual Koszul complex, which we can associate to each one of the rows of the matrix $\left(e_{i j}\right)$.

It is, however, of interest to consider the notion of syzygy we can associate to such a matrix $\left(e_{i j}\right)$. We say that

$$
\left(a_{1}, \ldots, a_{q}\right) \in R^{q}
$$


is in the first syzygy of the matrix $\left(e_{i j}\right)$ if

$$
\sum_{j=1}^{q} e_{i j} a_{j}=0
$$

for any $i=1, \ldots, s$. One also says that $\left(a_{1}, \ldots, a_{q}\right)$ belongs to the module of relations of the rows of $\left(e_{i j}\right)^{t}$. As it is easy to imagine, the explicit computation of the generators for such a module is a tremendous task, at least in the most general situation, but once again the Koszul complex can be of some use; indeed the first steps of the complex (see the explicit construction given in [16]) look like

$$
\cdots \rightarrow K_{2} \stackrel{d_{2}}{\longrightarrow} K_{1} \stackrel{d_{1}}{\longrightarrow} K_{0} \longrightarrow 0
$$

where $K_{0}=R^{s}, K_{1}=R^{q}, K_{2}=\Lambda^{s+1} R^{q}$. In this case $d_{1}=f$, while

$$
d_{2} \alpha=\left(d_{1}^{3} \circ \cdots \circ d_{1}^{s}\right) \alpha,
$$

for $\alpha \in K_{2}$ where $d_{1}^{j}, j=1, \ldots, s$, is the map $d_{1}$ associated to the (usual) Koszul complex of the $j-$ th row of $\left(e_{i j}\right)^{t}$.

Note that the order in which the rows of the matrix are considered is important, since

$$
d_{1}^{i} \circ d_{1}^{j}+d_{1}^{j} \circ d_{1}^{i}=0
$$

Also note that an explicit description of the map $d_{2}$ in the generalized Koszul complex can be given by just using the $s \times s$ minors of $\left(e_{i j}\right)$. Once again, $d_{1} \circ d_{2}=0$ and if one could prove the exactness of the Koszul complex, one would find a complete description for the module of relations of the matrix $\left(e_{i j}\right)^{t}$.

It has been proved in [6], [2] that a condition for the exactness of the Koszul complex can be given; such a condition, whose purpose is to generalize the notion of regular sequence, is however too restrictive and of very little practical use to us; on the other hand it is possible to show that, [6], if the matrix $\left(e_{i j}\right)$ is of maximal rank, then the generalized Koszul complex is exact.

In the next section we will need a weak version of the following algebraic condition on a matrix $\left(e_{i j}\right)$ of elements of $R$ : the importance of such a condition was pointed out in [11] and also by Malgrange as quoted in [20].

(EXT) Let $A=\left(e_{z j}\right)$ be a $q \times s$ matrix in $R, q \geq s$. For any $t \times q$ matrix $B$ in $R$ which generates the module of relations of the rows of $A$, $A$ generates the module of relations for the rows of $B$.

We can then prove the following result:

Theorem 2.1. Let $A=\left(e_{i j}\right)^{t}$ be a $q \times s$ matrix is $R, s \leq q$. For any $i=1, \ldots, s$, consider the $i \times i$ minors $M_{i k}$ obtained from $A$ by considering the first $i$ columns and the rows $1,2, \ldots, i-1, k$ (there are obviously $q-i+1$ such 
minors). Assume that, for any $i=1, \ldots, s$, the family $\left(M_{i k}\right)_{k=i, \ldots, q}$ is a regular sequence or that the ideal generated by $\left(M_{i k}\right)_{k=i, \ldots, q}$ is the whole ring $R$. Then A satisfies the condition (EXT).

Proof: To make the procedure more transparent, we will give the details for a $3 \times 2$ matrix; it is however clear how to extend the argument to a larger matrix. Let $\left(e_{i j}\right)$ be the transpose of the matrix $A ; i=1,2$ and $j=1,2,3$. Suppose $\alpha=\left(\alpha_{1}, \alpha_{2}, \alpha_{3}\right) \in R^{3}$ is in the module of relations of the rows of $A$ this means that

$$
\begin{aligned}
& \alpha_{1} e_{11}+\alpha_{2} e_{12}+\alpha_{3} e_{13}=0 \\
& \alpha_{1} e_{21}+\alpha_{2} e_{22}+\alpha_{3} e_{23}=0
\end{aligned}
$$

By the regularity hypothesis on $\left(e_{11}, e_{12}, e_{13}\right)$ and by the definition of the Koszul complex associated to the first row of $A$, we deduce from (2.3) that there is $\beta=\left(\beta_{1}, \beta_{2}, \beta_{3}\right) \in R^{3}$ such that

$$
\left\{\begin{array}{l}
\alpha_{1}=\beta_{1} e_{12}+\beta_{2} e_{13} \\
\alpha_{2}=-\beta_{1} e_{11}+\beta_{3} e_{13} \\
\alpha_{3}=-\beta_{2} e_{11}-\beta_{3} e_{12}
\end{array}\right.
$$

By inserting (2.5) into (2.4) we get

$$
\beta_{1} e_{12} e_{21}+\beta_{2} e_{13} e_{21}-\beta_{1} e_{11} e_{22}+\beta_{3} e_{13} e_{22}-\beta_{2} e_{11} e_{23}-\beta_{3} e_{12} e_{23}=0
$$

and now, if we set

$$
M_{12}=e_{11} e_{22}-e_{12} e_{21}, M_{13}=e_{11} e_{23}-e_{13} e_{21}, M_{23}=e_{12} e_{23}-e_{13} e_{22},
$$

equation $(2.6)$ becomes

$$
\beta_{1} M_{12}+\beta_{2} M_{13}+\beta_{3} M_{23}=0 .
$$

As we know, however, $M_{12}, M_{13}, M_{21}$ are not independent as they satisfy, from Laplace theorem applied to the matrix

$$
\left[\begin{array}{lll}
e_{11} & e_{12} & e_{13} \\
e_{21} & e_{22} & e_{23} \\
e_{11} & e_{12} & e_{13}
\end{array}\right],
$$

the following identity:

$$
e_{11} M_{23}-e_{12} M_{13}+e_{13} M_{12}=0 .
$$

Multiplying (2.7) by $e_{11}$, and using (2.8) we readily obtain

$$
M_{12}\left(\beta_{1} e_{11}-\beta_{3} e_{13}\right)+M_{13}\left(\beta_{2} e_{11}+\beta_{3} e_{12}\right)=0
$$


By the hypothesis on the regularity of the sequence $\left(M_{12}, M_{13}\right)$, we deduce that we can find $\lambda \in R$ such that

$$
\begin{aligned}
& \beta_{1} e_{11}-\beta_{3} e_{13}=\lambda M_{13} \\
& \beta_{2} e_{11}+\beta_{3} e_{12}=-\lambda M_{12}
\end{aligned}
$$

By replacing, in (2.10), (2.11), $M_{13}$ and $M_{12}$ by their development we obtain, respectively

$$
\left(\beta_{1}-\lambda e_{23}\right) e_{11}+\left(\lambda e_{21}-\beta_{3}\right) e_{13}=0
$$

and

$$
\left(\beta_{2}+\lambda e_{22}\right) e_{11}+\left(\beta_{3}-\lambda e_{21}\right) e_{12}=0 .
$$

From $(2.12)$, using the fact that $\left(e_{11}, e_{12}, e_{13}\right)$ is a regular sequence, we obtain that, for some $\sigma \in R$

$$
\beta_{1}=\lambda e_{23}+\sigma e_{13}
$$

and

$$
\beta_{3}=\lambda e_{21}+\sigma e_{11}
$$

Replacing (2.15) into (2.13) we deduce

$$
\beta_{2}=-\sigma e_{12}-\lambda e_{22}
$$

We can finally insert (2.14), (2.15) and (2.16) into (2.5), thus obtaining

$$
\left(\alpha_{1}, \alpha_{2}, \alpha_{3}\right)=\lambda\left(M_{23},-M_{13}, M_{12}\right) \text {. }
$$

So far, we have shown that the matrix $B$ which generates the module of relations of the rows of $A$ is the $1 \times 3$ matrix

$$
B=\left(M_{23},-M_{13}, M_{12}\right) .
$$

Let now $\gamma=\left(\gamma_{1}, \gamma_{2}, \gamma_{3}\right)$ be an element in the module of relations of $B$. This means that

$$
\gamma_{1} M_{23}-\gamma_{2} M_{13}+\gamma_{2} M_{12}=0 .
$$

Now, except for a change of sign and of order, (2.17) looks like (2.7), and therefore we can conclude that, for suitable $\lambda, \sigma \in R$, one has

$$
\left(\gamma_{1}, \gamma_{2}, \gamma_{3}\right)=\lambda\left(e_{21}, e_{22}, e_{23}\right)+\sigma\left(e_{11}, e_{12}, e_{13}\right)
$$


This concludes the proof that $A=\left(e_{i j}\right)$ satisfies (EXT). The reader will inmediately recognize how this proof carries over to larger matrices.

A matrix $A$ satisfying the conditions in Theorem 2.1 is said to be a regular matrix.

In the case in which $R$ is the polynomial ring $C\left[z_{1}, \ldots, z_{n}\right]$, the ring $\mathcal{O}$ of germs of holomorphic functions or the ring $H\left(\mathbb{C}^{n}\right)$ of entire functions, it is known (by the Nullstellensatz or by Cartan's Theorem B), that elements $P_{1}, \ldots, P_{q}$ generate all of $\mathbb{C}\left[z_{1}, \ldots, z_{n}\right], \mathcal{O}, H\left(\mathbb{C}^{n}\right)$ if and only if they have no common zeros. This however is not true if $R$ is a ring of holomorphic functions with growth conditions (such as the rings $H^{\infty}, A_{p}$, etc.).

Let us here point out a very simple example of a regular matrix; in $\mathbb{C}\left[z_{1}, z_{2}, z_{3}\right]$ we consider the matrix

$$
A^{t}=\left[\begin{array}{ccc}
z_{1} & z_{2} & z_{3} \\
z_{1}+1 & z_{2}+1 & z_{3}+1
\end{array}\right]
$$

it is immediate to check that Theorem 2.1 holds in this case.

In our propagation of regularity results, however, we will not need the full strength of the (EXT)-condition, but it will be sufficient, in general, to show the existence of a matrix $B$ (a $t \times q$ matrix in $R$ ) for which the sequence

$$
R^{s} \stackrel{A}{\rightarrow} R^{q} \stackrel{B}{\longrightarrow} R^{t}
$$

is exact. A good candidate for such a matrix, of course, would be (see Theorem 2.1) the matrix $B$ which generates the module of relations of the rows of the matrix $A$. In this case; Theorem 2.1 provides a condition, the regularity of some sequences, which ensures that (EXT) holds, and therefore (2.18) is true for $B$ the matrix which generates the modules of relations of the rows of $A$. In general, however, it may be difficult to construct explicitly such a matrix and, in any case, we may just use any $B$, as long as $(2.18)$ is exact, for our following results.

We note presently the following obvious fact, which we will need later on.

Proposition 2.1. Let $A$ be a $q \times s$ matrix in $R, q \geq s$, and let $B$ be any $t \times q$ matrix in $R$ which belongs to the module of relations of the rows of $A$. Then the following conditions are equivalent:

i) A generates the module of relations for the rows of $B$;

ii) the sequence (2.18)

$$
R^{s} \stackrel{A}{\longrightarrow} R^{Q} \stackrel{B}{\longrightarrow} R^{\ell}
$$


We therefore see that (EXT) is equivalent to the request that (2.18) is exact for $B$ the matrix which generates the module of relations of the rows of $A$.

One is therefore led to the investigation of conditions to ensure the exactness of (2.18). As it is easy to prove, if $A$ is a $q \times s$ matrix, $q \geq s$, one can easily show that a matrix $B$ for which (2.18) is a complex can be obtained by suitably setting up $\left(\begin{array}{c}q \\ s+1\end{array}\right)$ rows of $(s+1)$ minors of order $s$, each row being filled up with $q-(s+1)$ zeros. In particular, we can construct row matrices $B$ by just considering the $(s+1)$ minors of order $s$ which consist, e.g., of the first $(s+1)$ rows of $A$. If we call these minors $M_{I}$, for $|I|=s, I=\left(i_{1}, \ldots, i_{s}\right), 1 \leq i_{j} \leq s+1$, we set $M_{I}=M_{\mathrm{i}}$ for $i \in\{1, \ldots, s+1\} \backslash I$, and we have

$$
\sum_{i=1}^{s+1}(-1)^{i} e_{i 1} M_{i}=0
$$

therefore $B$ will look like

$$
B=\left(M_{1}, M_{2}, \ldots, M_{9+1}, \frac{q-(s+1)}{0,0, \ldots, 0)}\right.
$$

It is therefore sufficient to show that such a matrix is generated by the rows of $A$, and this follows in a ratural way from the second part of the proof of Theorem 2.1, under those same hypotheses.

As it will be cleas in the sequel, we will need $R$ to be either $\mathbb{C}\left[z_{1}, \ldots, z_{n}\right]$, the ring of polynomials in $\mathbb{C}^{n}$, or $H\left(C^{n}\right)$, the ring of entire functions. Actually, we will also need to take rings of holomorphic functions satisfying suitable growth conditions, but this aspect will be dealt with in the later sections.

Therefore we want to know when, for a given matrix $A$ in $R=\mathbb{C}\left[z_{1}, \ldots, z_{n}\right]$ or $H\left(C^{n}\right)$ one can find a matrix $B$ such that (2.18) is exact. The following important fact will help us:

Proposition 2.2. A sequence

$$
R^{s} \stackrel{A}{\longrightarrow} R^{q} \stackrel{B}{\longrightarrow} R^{t}
$$

with $A, B$ polynomial matrices is exact for $R=\mathbb{C}\left[z_{1}, \ldots, z_{n}\right]$ if and only if it is exact for $R=H\left(\mathbb{C}^{n}\right)$.

Proof: This is immediate, in view of the well known fact that the ring $H\left(\mathbb{C}^{n}\right)$ is faithfully flat over the ring $C\left[z_{1}, \ldots, z_{n}\right]$, see [1].

In [1], Andreotti and Nacinovich devote much effort to the study of the exactness of (2.18), with, among other things, the purpose of understanding the work of Ehrenpreis and Malgrange on the solvability of systems of differential equations. In particular, in [1], they prove the following characterization of those matrices $A$ for which there exists $B$ such that $(2,18)$ is exact: 
Proposition 2.3. Given a matrix $A$, there exists $B$ such that the sequence (2.18) is exact if any only if the cokernel of the map induced by $A$ has no torsion.

An immediate consequence is the following

Proposition 2.4. If $A$ is a regular matrix, then coker $(A)$ has no torsion.

Proof: This follows immediately from Theorem 2.1 and Proposition 2.3.

The reader may notice that while the condition of regularity for a matrix may be stronger than the no-torsion condition, it is certainly easier to verify.

Finally we note what happens in the case in which $s=1$ (the case which, essentially, was dealt with by Ehrenpreis in $(10])$ :

Proposition 2.5. Let $P_{1}, \ldots, P_{q}$ be polynomials in $\mathbb{C}^{n}$, and let $A$ be the matrix $\left(P_{1}, \ldots, P_{\ell}\right)^{t}$. Then the following conditions are equivalent:

i) coker $(A)$ has no torsion;

ii) the matrix $B$, whose $\left(\begin{array}{l}q \\ 2\end{array}\right)$ rows are $P_{i} e_{j}-P_{j} e_{i}$, for $e_{i}=(0,0, \ldots, 1, \ldots, 0)$ makes (2.18) an exact sequence;

iii) $P_{1}, \ldots, P_{q}$ have no common factors.

\section{Proof:}

iii) $\Rightarrow$ ii) Indeed if $\vec{h}=\left(h_{1}, \ldots, h_{q}\right)^{t}$ is such that $B \cdot \vec{h}=0$, we deduce (by the construction of $B$ ) that

$$
P_{i} h_{j}-P_{j} h_{i}=0
$$

and therefore the function

$$
g=\frac{h_{i}}{P_{i}}=\frac{h_{j}}{P_{j}}=\ldots
$$

is entire (as codim $\left\{z \in \mathbb{C}^{n}: P_{1}(z)=\cdots=P_{q}(z)=0\right\} \geq 2$ by hypothesis) and therefore a polynomial (as a quotient of polynomials). It follows that $\vec{h}=\left(P_{1} g, \ldots, P_{q} g\right)^{t}=A \cdot g$.

ii) $\Rightarrow$ i) By Proposition 2.3 .

ii) $\Rightarrow$ iii) Indeed, suppose $P_{1}, \ldots, P_{q}$ have a common factor $Q \not \equiv 1$, so that $P_{j}=Q P_{j}^{\prime}$. Then the $q$-tuple $\vec{P}^{\prime}=\left(P_{1}^{\prime}, P_{2}^{\prime}, \ldots, P_{q}^{\prime}\right)^{t}$ satisfies $B \cdot \vec{P}^{\prime}=0$ but there is no $g$ such that $A \cdot g=\vec{P}^{\prime}$.

i) $\Rightarrow$ iii) This follows as the previous step, by recalling that

$$
\text { coker } A=\frac{(\mathbb{C}[z])^{\natural}}{A \cdot \mathbb{C}[z]}
$$


and $\vec{h}=\left(h_{1}, \ldots, h_{q}\right)^{t} \in(\mathbb{C}[z])^{q}$ is in the torsion of coker $(A)$ if and only if there exists $Q \in \mathbb{C}[z]$, such that $Q \cdot \vec{h} \in A \cdot \mathbb{C}[z]$, with $Q$ not in the ideal generated by $A$.

\section{Division Theorems}

For the problems we will be dealing with in this section, we need some technical results on the division of holomorphic functions with growth conditions. Such resuits have a long history as they go back to the first approaches to the surjectivity problem for constant coefficients partial differential operators and for convolution operators.

Let us start with the classical Ehrenpreis-Malgrange lemma, which is usually needed to control the growth of a quotient of an entire function by a polynomial [11]:

Theorem 3.1. Let $P(z)=a_{m}\left(z_{1}, \ldots, z_{n-1}\right) z_{n}^{m}+\cdots+a_{0}\left(z_{1}, \ldots, z_{n-1}\right)$ be a polynomial in $n$ variables and let $F(z)$ be a holomorphic function in $\left|z_{j}-z_{j}^{0}\right| \leq \delta$ for all $j=1, \ldots, n$, where $z^{0}=\left(z_{1}^{0}, \ldots, z_{n}^{0}\right)$ is some fixed point. If $|P(z) F(z)| \leq$ $C$ in $\left\{z \in \mathbb{C}^{n}:\left|z_{j}-z_{j}^{0}\right| \leq \delta\right.$ for all $\left.j\right\}$, then there are constants $A, B>0$, only depending on $P$, such that

$$
\left|F\left(z^{0}\right)\right| \leq A C \delta^{-B}
$$

This result can be extended to the case of non-principal polynomial ideals. In this case, the framework is the following: let $P_{1}, \ldots, P_{q}$ be polynomials in $\mathbb{C}^{n}$ and assume that $F$ is an entire function which belongs to the ideal $I_{H}$ generated by $P_{1}, \ldots, P_{q}$ in $H\left(\mathbb{C}^{n}\right)$. This means that there are entire functions $\lambda_{1}, \ldots, \lambda_{q} \in H\left(\mathbb{C}^{n}\right)$ such that

$$
F=\lambda_{1} P_{1}+\cdots+\lambda_{q} P_{q}
$$

the problem which one wants to address is the following: suppose $F$ satisfies some additional growth conditions (e.g. $F$ is of exponential type, or $F$ is the Fourier transform of a completely supported distribution, etc.); can we deduce the same growth on the $\lambda_{i}$ 's? This is certainly false in general, but more precisely, we may ask whether from (3.1) we can deduce the existence of $\tilde{\lambda}_{1}, \ldots, \tilde{\lambda}_{q}$ such that

$$
F=\tilde{\lambda}_{1} P_{1}+\cdots+\tilde{\lambda}_{q} P_{q}
$$

and such that they satisfy the same growth of $F$. In other words, suppose $F \in A$, where $A$ is a topological vector space of entire functions satisfying suitable growth conditions: it is true that

$$
I_{A}=I_{H} \cap A,
$$


where $I_{A}=\left\{f \in H\left(\mathbb{C}^{n}\right): f=\varphi_{1} P_{1}+\cdots+\varphi_{q} P_{q}, \varphi_{j} \in A\right\}$ ?

A very complete theory in this direction has been developed by Ehrenpreis in [11], as a necessary step towards the proof of his celebrated Fundamental Principle. Actually, Ehrenpreis employed Theorem 3.1 (essentially the case in which $q=1$ ), together with some Oka-Cartan vanishing of cohomology, to prove that (3.2) is true for a very large class of spaces $A$, which arise as Fourier transform of the dual of Analytically Uniform spaces.

We refer to [11] for the definition of Analytically Uniform spaces, and we content ourselves with the mention of a few examples.

Example 3.1. The following spaces are Analytically Uniform: the space $\mathcal{E}(\Omega)$ of $C^{\infty}$ functions on an open convex subset $\Omega$ of $\mathbb{R}^{n}$, the space $\mathcal{D}^{\prime}(\Omega)$ of Schwartz distributions on an open convex subset $\Omega$ of $\mathbb{R}^{n}$, the spaces $\mathcal{E}_{B}(\Omega), \mathcal{D}_{B}^{\prime}$ $(\Omega)$ of Beurling ultradifferentiable functions and of Beurling ultradistributions (provided that $B$ satisfies some natural conditions), the space $H(\Omega)$ of holomorphic functions on an open convex subset $\Omega$ of $\mathbb{C}^{n}$. For all these spaces one can consider a (strong) dual space $\left(\mathcal{E}^{\prime}, \mathcal{D}, \mathcal{E}_{B}^{\prime}, \mathcal{D}_{B}, H^{t}\right.$, etc.) and, on the duals, a Fourier (or Fourier-Borel) transform can be defined. Then, respectively, the space $A$ will be described as follows: $\mathcal{E}^{\prime}(\Omega)=\left\{F \in H\left(\mathbb{C}^{n}\right): \exists K \subset \Omega\right.$ compact convex, $A, B>0:|F(z)| \leq A(1+|z|)^{B} \exp \left(H_{K}(\right.$ Imz $\left.\left.)\right)\right\}$ for $H_{K}$ the supporting function of $K ; \hat{\mathcal{D}}(\Omega)=\left\{F \in H\left(\mathbb{C}^{n}\right): \exists K \subset \Omega\right.$ compact convex, and for any $B>0$ there is $\left.A>0:|F(z)| \leq A(1+|z|)^{-B} \exp \left(H_{K}(\operatorname{Im} z)\right)\right\}$; and $\hat{H}^{\prime}(\Omega)=$ $\left\{F \in H(\Omega): \exists K \subset \Omega\right.$ compact convex, $\left.A>0:|F(z)| \leq A \exp \left(H_{K}(z)\right)\right\}$. Note that when $\Omega$ is, respectively, $R^{n}$ or $C^{n}$, the spaces which we have described are, also, algebras under pointwise multiplication.

We can now state Ehrenpreis' division theorem for Analytically Uniform Spaces (we refer to [11] for its original proof):

Theorem 3.2. Let $P_{1}, \ldots, P_{q}$ be polynomials in $\mathbb{C}^{n}$, and let $A$ be a topological vector space which is isomorphic to the space of Fourier transforms of an Analytically Uniform space. Let $F \in A$ be such that, for some $\lambda_{1}, \ldots, \lambda_{q} \in H\left(\mathbb{C}^{n}\right)$,

$$
F=\lambda_{1} P_{1}+\cdots+\lambda_{q} P_{q}
$$

then one can find $\tilde{\lambda}_{1}, \ldots, \tilde{\lambda}_{q} \in$ A such that

$$
F=\tilde{\lambda}_{1} P_{1}+\cdots+\tilde{\lambda}_{q} P_{q},
$$

i.e. (3.2) is true.

As it will become apparent in section 4 , the polynomials $P_{1}, \ldots, P_{q}$ appear as symbols of suitable linear constant coefficients partial differential operators; therefore, in connection with the study of infinite order differential operators, as well as of convolution operators, one may wish to consider ideals generated by entire functions, instead of polynomials. In this case, however, one cannot 
prove any theorem such as Theorem 3.2, uniess some restrictive hypotheses are asked on $\left(P_{1}, \ldots, P_{q}\right)$. These hypotheses, in particular, always require that the varieties

$$
\left\{z \in \mathbb{C}^{n}: P_{1}(z)=\cdots=P_{j}(z)=0\right\}, j=1, \ldots, q
$$

are of codimension $j$, and go under the name of "slowly decreasing" conditions.

We will need, in section 4 , two such division theorems. The first was originally proved in [15], to which we refer the reader for the proof and the necessary definitions.

Theorem 3.3. Let $P_{1}, \ldots, P_{q}$ be entire functions of exponential type zero (also known as entire functions of infraexponential type). Let $\hat{\mathcal{B}}_{K}$ denote, for $K \subset \mathbb{P}^{n}$ a compact set, the space of Fourier transforms of all hyperfunctions on $\mathbb{R}^{n}$, supported by $K$. Then if $\left(P_{1}, \ldots, P_{q}\right)$ is slowly decreasing we have

$$
I_{\dot{\mathcal{B}}_{K}}=I_{H} \cap \hat{\mathcal{B}}_{K}
$$

As for the second division theorem which we need, we have to consider division in $\hat{\mathcal{D}}(K)$; unfortunately, no such a result has ever been explicitly given in the literature (although it is somehow implicit in [3], [4]) and therefore we will need to give a proof here.

In order to prove the propagation of regularity theorem, as it has been shown in [23], we need to have the following division result: let $K, T, \bar{K}$ be compact convex sets in $\mathbb{R}^{n}$ with $K=\tilde{K}-T$. Let $F \in \tilde{\mathcal{D}}(\tilde{K})$ and $g_{1}, \ldots, g_{r} \in \hat{\mathcal{E}}^{\prime}(T)$. Suppose that, on $\mathbb{C}^{n}$

$$
F=\lambda_{1} g_{t}+\cdots+\lambda_{r} g_{r}
$$

for some $\lambda_{1}, \ldots, \lambda_{r} \in H\left(\mathbb{C}^{n}\right)$; we look for conditions on $\left(g_{1}, \ldots, g_{r}\right)$ so that the $\lambda_{i}$ 's can be replaced by $\tilde{\lambda}_{1}, \ldots, \tilde{\lambda}_{r} \in \hat{\mathcal{D}}(K)$ such that, on $\mathbb{C}^{n}$,

$$
F=\tilde{\lambda}_{1} g_{1}+\cdots+\tilde{\lambda}_{r} g_{r}
$$

Note that this division result is different from those which we already quoted, because $T \neq\{0\}$ and therefore the growth of $F$ and that of $\tilde{\lambda}_{1}, \ldots, \tilde{\lambda}_{r}$ do not coincide.

In order to prove such a division result, we need to start with the case in which the variety

$$
V=\left\{z \in \mathbb{C}^{n}: g_{1}(z)=\cdots=g_{r}(z)=0\right\}
$$

is discrete, and we therefore assume also $r=n$. The following hypothesis is needed on the $n$-tuple $\left(g_{1}, \ldots, g_{n}\right)$ :

Deffnitions 3.1 We say that $g=\left(g_{1}, \ldots, g_{n}\right)$ is $T$-slowly decreasing if the following conditions are satisfied: 
$(\alpha)$ for any $\varepsilon>0$ there exists constants $A, B>0$ such that the connected components of the set

$$
S(g ; A, B, \varepsilon)=\left\{z \in \mathbb{C}^{n}: d\left(z, V_{i}\right) \leq A(1+|z|)^{-B} \exp (-\varepsilon|\operatorname{Imz}|)\right\}
$$

are relatively compact and with uniformly bounded dianeters, for $d\left(z, V_{i}\right)=$ $\min \left(1\right.$, distance from $z$ to $\left.V_{i}\right), V_{i}=\left\{z \in \mathbb{C}^{n}: g_{i}(z)=0\right\}$;

$(\beta)$ there are positive constants $C, D, m$ such that

$$
\begin{aligned}
& \left|g_{i}(z)\right| \geq C(1+|z|)^{-D} d\left(z, V_{i}\right)^{m} \exp \left(H_{T}(\operatorname{Im} z)\right), \\
& \left(\sum\left|g_{i}(z)\right|^{2}\right)^{1 / 2}=|g(z)| \geq C(1+|z|)^{-D} d(z, V)^{m} \exp \left(H_{T}(\operatorname{Imz})\right) .
\end{aligned}
$$

Remark. In the literature, the expression "slowly decreasing" describes a large number of similar (but not identical) conditions. All of them look essentially the same, except for the necessary adjustments which different weights compel to introduce and, roughly speaking, all these conditions require $|g(z)|$ not to be too small too often. Concrete examples of $T$-slowly decreasing $n$ tuples can be found in [3].

We need to quote from [4] a basic interpolation result: let $g_{1}, \ldots, g_{n}$ be analytic functions such that $V=\left\{z \in \mathbb{C}^{n}: g_{1}(z)=\cdots=g_{n}(z)=0\right\}$ is discrete, and let $\Omega=\Omega(\varepsilon)$ be a bounded component of the analytic polyhedron

$$
P(\varepsilon)=\left\{z \in \mathbb{C}^{n}:\left|g_{i}(z)\right|<\varepsilon_{i}, 1 \leq i \leq n\right\},
$$

where $\varepsilon=\left(\varepsilon_{1}, \ldots, \varepsilon_{n}\right)$ is chosen so that $P(\varepsilon)$ is nondegenerate. It is always possible to choose functions $Q_{i j}$ on $\bar{\Omega} \times \bar{\Omega}$ such that

$$
g_{i}(\zeta)-g_{j}(z)=\sum_{j=1}^{n} Q_{i j}(\zeta, z)\left(\zeta_{j}-z_{j}\right) i=1, \ldots, n,
$$

and we set

$$
H(\zeta, z)=\operatorname{det}\left[Q_{i j}(\zeta, z)\right]
$$

The following result is given in [4]:

Theorem 3.4. Let $\mathcal{O}=\left\{z \in \Omega:\left|g_{i}(z)\right|<\frac{1}{2} \eta_{i}, 1 \leq i \leq n\right\}$ and let $F$ be analytic on $\bar{\Omega}$ such that $F(z)=f_{1} g_{1}+\cdots+f_{n} g_{n}$ for some $f_{1}, \ldots, f_{n} \in H(\bar{\Omega})$. If $\eta=\prod_{i=1}^{n} \eta_{i}, \Gamma$ denotes the distinguished boundary of $\Omega$, and

(i) $|F(z)| \leq M$ on $\bar{\Omega}$,

(ii) $|H(\zeta, z)| \leq D$ on $\bar{\Omega} \times \mathcal{O}$,

(iii) $\int_{\Gamma}\left|d \zeta_{\Omega} \wedge \cdots \wedge d \zeta_{n}\right| \leq L$

(iv) $\left|g_{i}(\zeta)\right| \leq D_{1}$ on $\bar{\Omega}$ 
then we can find $\alpha_{1}, \ldots, \alpha_{n} \in H(\Omega)$ such that

$$
F(z)=\alpha_{1}(z) g_{3}(z)+\cdots+\alpha_{n}(z) g_{n}(z) \text { on } \Omega
$$

and $\left|\alpha_{i}(z)\right| \leq \frac{C D M L D_{1}^{n-2}}{\eta^{2}}$ on $\mathcal{O}$, for $C$ a constant only depending on $n$.

The interest of this result is due to the fact that one can employ it to (semilocally) control the bounds of holomorphic functions. Its proof is by no means trivial, as it relies on the construction of an interpolation formula (called in [4] the Jacobi interpolation formula) which only applies to discrete varieties (but we shall soon see that non-discrete varieties can be considered as well).

Theorem 3.5. Let $K, T, \tilde{K}$ be compact convex sets in $\mathbb{R}^{n}$, with $K=\tilde{K}-T$. Let $F \in \hat{\mathcal{D}}(\tilde{K})$ and suppose $g_{1}, \ldots, g_{n} \in \hat{\mathcal{E}}^{\prime}(T)$ are such that $\left(g_{1}, \ldots, g_{n}\right)$ is $T$. slowly decreasing. Then if there exist entire functions $f_{1}, \ldots, f_{n} \in H\left(\mathbb{C}^{n}\right)$ such that

$$
F(z)=f_{1}(z) g_{1}(z)+\cdots+f_{n}(z) g_{n}(z), z \in \mathbb{C}^{n},
$$

one can find entire functions $\lambda_{1}, \ldots, \lambda_{n} \in \hat{\mathcal{D}}\left(K_{\varepsilon}\right)$ such that

$$
F(z)=\lambda_{1}(z) g_{1}(z)+\cdots+\lambda_{n}(z) g_{n}(z), z \in \mathbb{C}^{n}
$$

where $K_{\varepsilon}$ is the $\varepsilon$-neighborhood of $K, K_{\varepsilon}=\left\{z \in \mathbb{C}^{n}: d(z, K) \leq \varepsilon\right\}$.

Proof: The main ideas of this proof have already been given in [3], [4], but one still has to be careful with the bounds. To begin with, we exploit the notion of $T$-slow decrease to find constants $A, B>0$ so that Theorem 3.4 can be applied to each connected component $\Omega$ of $S(g ; A, B, \varepsilon)$, and, by $(\beta)$ in the definition of slow decrease, we can show that, for any $z \in \bar{\Omega}$, and for $\Gamma$ the distinguished boundary of $\Omega$,

$$
\int_{\Gamma}\left|d \zeta_{1} \wedge \cdots \wedge d \zeta_{n}\right| \leq A_{1}(1+|z|)^{B_{\mathfrak{\imath}}} \exp (\varepsilon|\operatorname{Imz}|)
$$

for some $A_{1}, B_{1}>0$. We can now easily apply Theorem 3.4 in which, now, we can take the following values for the constants: $M=A^{\prime}(1+|z|)^{-B^{\prime}} \exp \left(H_{\bar{k}}(\operatorname{Imz})\right)$, for any $B^{\prime}>0$ and some $A^{\prime}>0 ; D=A^{\prime \prime}(1+|z|)^{B^{\prime \prime}} \exp \left(H_{n T}(\operatorname{Imz})\right)$ for some $A^{n}, B^{\prime \prime}>0 ; D_{1}=\hat{A}(1+|z|)^{\dot{B}} \exp \left(H_{T}(\operatorname{Im} z)-\varepsilon|\operatorname{Im} z|\right)$, for some $\hat{A}, \hat{B}>0$; $\eta=A(1+|z|)^{-B} \exp \left(H_{n t}(\operatorname{Im} z)-n m \varepsilon|\operatorname{Imz}|\right)$. From these explicit bounds we deduce, via Theorem 3.4, that we can find functions $\alpha_{1}, \ldots, \alpha_{n}$, analytic in $S(g ; A, B, \varepsilon)$, such that

$$
F(z)=\alpha_{1}(z) g_{1}+\cdots+\alpha_{n}(z) g_{n}(z) \text { on } S\left(g ; A^{*}, B^{*}, \varepsilon\right)
$$

for some $A^{*}, B^{*}>0$, and such that, on $S\left(g ; A^{*}, B^{*}, \varepsilon\right)$

$$
\left|\alpha_{i}(z)\right| \leq \tilde{A}(1+|z|)^{-\bar{B}} \exp \left(H_{K_{e}}(\operatorname{Im} z)\right)
$$


where $K_{\varepsilon}$ can be made as small as desired, around $K$, by taking $\varepsilon$ sufficiently small for all $\tilde{B}>0$ and some $\tilde{A}>0$. Note that the constants which appear in the bounds do not depend on the choice of the component $\Omega$ of $S(g ; A, B)$, in view of $(\alpha)$ above. The proof can now be concluded by extending in a $C^{\infty}$ fashion the $\alpha_{1}$ 's to all of $\mathbb{C}^{n}$, via a Whitney cutoff function, and then by modifying the result from $C^{\infty}$ to holomorphic in a standard way with a diagram chase on the Koszul complex associated to $g$, as it is shown in detail in [2], [3], [14].

Theorem 3.4 is all we shall need in the sequel but, of course, the $\varepsilon$-neighborhood $K_{\epsilon}$ of $K$ can be easily replaced by $K$ (i.e. we can get a stricter control on the bounds) if the definition of $T$-slow decrease in strengthened accordingly.

Let us now describe the general ideas which can enable us to extend Theorem 3.4 to the general (i.e. non discrete) case; the procedure which we need to follow is pretty well known, by now, and we do not wish to describe all the details; it is however of some interest to describe the necessary steps and, therefore, how the slowly decreasing condition must be modified.

As we mentioned before, in order to apply the Jacobi interpotation formula, which provides us with the explicit bounds in Theorem 3.4, it is essential to be dealing with a discrete variety. Thus, when looking at an ideal generated by $g_{1}, \ldots, g_{r}, 1 \leq r<n, g_{j} \in \hat{\mathcal{E}}^{\prime}(T)$, we need to devise a way to reduce the problem to the discrete situation. This situation has already been discussed in several instances (see, e.g., [3], [4], [15], etc.) and a "standard" argument can now be given. The idea consists in "cutting" the variety

$$
V=\left\{z \in \mathbb{C}^{n}: g_{1}(z)=\cdots=g_{r}(z)=0\right\}
$$

which is assumed to be a complete intersection (actually, even though this is never explicitely mentioned in the previous works, every variety $\left\{z \in \mathbb{C}^{n}\right.$ : $\left.g_{1}(z)=\cdots=g_{j}(z)=0\right\}, j=1, \ldots, r$, needs to be a complete intersection) by a family $\mathcal{L}=\{L\}$ of $r$-dimensional affine complex spaces; the resulting intersections are discrete and if one has a slowly descreasing hypothesis on each $L$, then one can apply the Jacobi interpolation formula on every section, and they try to glue together the results. For this approach to work, we clearly need two basic conditions; first we require that the spaces $L$ inside the family $\mathcal{L}$ change in an analytic fashion, so that also the interpolation obtained via the different Jacobi formulas is analytic; second we need to have some uniformity in the constants which appear on every space $L$. This informal discussion can be summed up by the following definitions:

Definition 3.2. Let $g_{1}, \ldots, g_{\mathrm{r}} \in \hat{\mathcal{E}}^{\prime}(T)$ for $T$ some compact convex subset of $\mathbb{R}^{n}$. We say that $g=\left(g_{1}, \ldots, g_{r}\right)$ is $T$-slowly decreasing if there exists a family $\mathcal{L}=\{L\}$ of $r$-dimensional affine complex spaces, whose union covers $\mathbb{C}^{n}$, such that, for suitable constants $C, D, m>0$ one has, for all $L \in \mathcal{L}$ (and with the 
same notations used before),

$$
\begin{aligned}
\left|g_{i}(z)\right| & \geq C(1+|z|)^{-D} d\left(z, V_{i} \cap L\right)^{m} \exp \left(H_{T}(\operatorname{Im} z)\right), z \in L, \\
|g(z)| & \geq C(1+|z|)^{-D} d(z, V \cap L)^{m} \exp \left(H_{T}(\operatorname{Imz})\right), z \in L .
\end{aligned}
$$

Moreover, for all $\varepsilon>0$, there are constants $A, B>0$ such that, for all $L \in \mathcal{L}$, the sets

$$
\mathcal{O}_{L}(g ; A, B, \varepsilon)=\left\{z \in L: d\left(V_{i} \cap L\right) \leq A(1+|z|)^{-B} \exp \{-\varepsilon|\operatorname{Im} z|), \forall i\right\}
$$

have relatively compact connected components, whose diameters are uniformly bounded.

To actually prove a division theorem such as Theorem 3.5 , we need to apply a vanishing of cohomology argument to a suitably chosen covering of $C^{n}$. Let us then fix $L \in L, \varepsilon, A, B$ so that any connected component $G$ of $\mathcal{O}_{L}(g ; A, B, \varepsilon)$ has its diameter bounded by some fixed constant. An open set $\Omega \subseteq \mathbb{C}^{n}$ is said to be good if it has the form

$$
\Omega=\left\{z \in \mathbb{C}^{n}: \exists \zeta \in G \text { with }|z-\zeta| \leq \alpha(1+|\zeta|)^{-\beta} \exp \left(-H_{K}(\operatorname{Im} \zeta)\right)\right\},
$$

for some positive constants $\alpha, \beta$. By fixing $g, L, \varepsilon, A, B, \alpha, \beta$ we obtain what is said to be a good family of sets, which we denote by $\mathcal{C}$. Finaliy, a good refinement $\mathcal{C}^{\prime}$ of $\mathcal{C}$ is any family obtained by decreasing $A, \alpha$, and increasing $B, \beta, \varepsilon$; note that for any good refinement $\mathcal{C}^{\prime}$ of $\mathcal{C}$, there is a naturally defined refinement map $\rho: \mathcal{C}^{\prime} \rightarrow \mathcal{C}$.

Definition 3.3. The family $\mathcal{L}$ is said to be almost parallel if, given a good family $\mathcal{C}$, there exists a good refinement $\mathcal{C}^{t}$ of $\mathcal{C}$ such that, whenever $\Omega_{0}, \Omega_{1} \in \mathcal{C}^{\prime}$, it is

$$
\Omega_{0} \cap \Omega_{1} \neq \phi \Rightarrow \bar{\Omega}_{0} \cup \vec{\Omega}_{1} \subseteq \rho\left(\Omega_{0}\right) \cap \rho\left(\Omega_{1}\right) .
$$

Deffnition 3.4. The family $\mathcal{L}$ is said to be analytic if there is a good family $\mathcal{C}$, associated to $\mathcal{L}$, with the following property: given $\Omega \in \mathcal{C}$, associated to a space $L \in \mathcal{L}$, there are analytic local coordinates $(s, t)$ on $\Omega$ such that

$$
\Omega \cap\{(s, t): t=0\}=\Omega \cap L
$$

and

$$
\Omega \cap\{(s, t): t=\text { const }\}=\Omega \cap L_{t},
$$

for some $L_{t} \in \mathcal{L}$.

With the use of these definitions, and with arguments similar to those used for the proof of Theorem 3.5 (but see also Theorems 9, 10, 11 in [3]), we can prove our main division result: 
Theorem 3.6. Let $g_{3}, \ldots, g_{r} \in \hat{\mathcal{E}}^{\prime}(T), T$ compact convex in $\mathbb{R}^{n}$, be $T$-slowly decreasing with respect to an analytic almost parallel family of lines. Let $F \in$ $\hat{\mathcal{D}}(\tilde{K}), \bar{K}=K+T$ a convex compact subset of $\mathbb{R}^{n}$, be such that, for some entire functions $f_{1}, \ldots, f_{r} \in H\left(\mathbb{C}^{n}\right)$,

$$
F(z)=f_{1}(z) g_{1}(z)+\cdots+f_{r}(z) g_{r}(z), z \in \mathbb{C}^{n} .
$$

Then one can find entire functions $\lambda_{1}, \ldots, \lambda_{r} \in \hat{\mathcal{D}}\left(K_{\varepsilon}\right)$, where $K_{\varepsilon}$ is an $\varepsilon$ neighborhood of $K$, and $\varepsilon$ can be taken as small as desired, such that

$$
F(z)=\lambda_{1}(z) g_{1}(z)+\cdots+\lambda_{r}(z) g_{\tau}(z), z \in \mathbb{C}^{n} .
$$

To conclude this section we notice that many examples of $T$-slowly decreasing $r$-tuples can be constructed by employing exponential polynomials and exponential sums, as it is shown in [3], [4].

\section{Propagation of regularity}

In this final section we merge the algebraic results from section 2 with the division theorems of section 3, to provide several removability of singularity theorems for solutions of rectangular systems of suitable equations. All of these results reduce to the famous Hartogs' removability of singularity theorem, when the system which one considers is the Cauchy-Riemann system. The first result we wish to prove was, essentially, stated by Ehrenpries in [11], but not proof was given, and the hypotheses were somehow difficult to describe, so that it is probably worthwhile to give a new treatment of such a phenomenon:

Definition 4.1. A $q \times x$ matrix $A$ in a ring $R$ is said to be strongly regular if it is regular and, moreover, the following is true: if, for $j=1, \ldots, s-1, A_{j}$ is the matrix deduced by $A$ by only considering the first $j$ rows, then $A_{j}^{t}$ is regular. Moreover, we require that the $q-s+1$ minors of order $s$ of $A$ formed with the first $s-1$ rows of $A$ form a regular sequence.

Theorem 4.1. Let $P=\left[P_{i j}\right]$ be a $q \times x$ matrix of polynomials in $\mathbb{C}^{n}, 1 \leq s \leq$ $q, q \geq 2$. Let $P_{i j}(D)$ denote the differential operator (inear and with constant coefficients) obtained from the polynomial $P_{i j}$ by replacing $z=\left(z_{1}, \ldots, z_{n}\right)$ by $D=\left(-i \frac{\partial}{\partial x_{1}}, \ldots,-i \frac{\partial}{\partial x_{n}}\right)$, for $x=\left(x_{1}, \ldots, x_{n}\right) \in \mathbb{R}^{n}$. Let $\Omega \subseteq \mathbb{R}^{n}$ be bounded and convex, $n \geq 2$, and suppose $K \subseteq \Omega$ be compact and convex. Then if $\vec{f}=\left(f_{1}, \ldots, f_{s}\right), f_{j} \in \mathcal{E}(\Omega \backslash K)$, satisfies, in $\Omega \backslash K$,

$$
\sum_{j=1}^{s} P_{i j}(D) f_{j}=0, i=1, \ldots, q
$$

and $\varepsilon>0$, one can find a unique element $\vec{f}^{*}=\left(f_{1}^{*}, \ldots, f_{s}^{*}\right), f_{j}^{*} \in \mathcal{E}(\Omega)$ such that

$$
\sum_{j=1}^{s} P_{i j}(D) f_{j}^{*}=0, i=1, \ldots, q, \text { on all of } \Omega
$$


and $f_{j}^{*}$ coincides with $f_{j}$ on $\Omega \backslash K_{\varepsilon}$, for $K_{\varepsilon}$ an $\varepsilon$-neighborhood of $K$.

Proof: As for the case of Theorem 3.2, we will make our proof more transparent, by describing it in detail in the case in which $P$ is a $3 \times 2$ matrix. The general case can be treated in exactly the same way. Suppose, therefore, that $\vec{f}$ satisfies (4.1). By allowing a small modification near $\partial K$ we can find $\tilde{f}_{j} \in \mathcal{E}(\Omega)$ such that $\tilde{f}_{j}=f_{j}$ on some $\Omega \backslash K_{\varepsilon}$; set

$$
g_{i}=\sum_{j=1}^{s} P_{i j}(D) \tilde{f}_{j} \quad, \quad i=1, \ldots, q .
$$

Because of (4.1), and by the definition (4.2), one has that

$$
g_{i} \in \mathcal{D}\left(K_{\varepsilon}\right), \quad i=1, \ldots, q .
$$

Moreover, if $Q=\left[Q_{j t}(D)\right]$ is a matrix which generates the module of relations of the rows of $P$ (or, as we saw, any matrix which generates a module properly contained in such module of relations so that $Q \circ P=0$ ), we have that

$$
\sum_{t=1}^{q} Q_{j t}(D) g_{t}=0
$$

Since the $g_{i}$ 's have compact support, we can take the Fourier transform of (4.3) which gives us

$$
\sum_{t=1}^{q} Q_{j t} \cdot \hat{g}_{t}=0
$$

By the hypothesis of strong regularity on the matrix $P$, and by the faithful flatness of $H\left(\mathbb{C}^{n}\right)$ over $\mathbb{C}\left[z_{1}, \ldots, z_{n}\right]$, we deduce the existence of entire functions $k_{1}, \ldots, k_{s}$ such that

$$
\hat{g}_{2}=\sum_{j=1}^{s} P_{i j} k_{j} \quad i=1, \ldots, q
$$

To explicitly see how (4.5) can be used, we now set $s=2, q=3$. We then have

$$
\left\{\begin{array}{l}
P_{11} k_{1}+P_{12} k_{2}=\hat{g}_{1} \\
P_{21} k_{1}+P_{22} k_{2}=\hat{g}_{2} \\
P_{31} k_{1}+P_{32} k_{2}=\hat{g}_{3} .
\end{array}\right.
$$

By applying Theorem 3.2 to the first equation in (4.6) we deduce the existence of $h_{1}, h_{2} \in \hat{\mathcal{D}}\left(K_{\varepsilon}\right)$ such that

$$
P_{11} h_{1}+P_{12} h_{2}=\hat{g}_{1} .
$$


Note that, by the regularity of the sequence $\left(P_{11}, P_{12}\right)$, we know that

$$
h_{1}=k_{1}+\rho P_{12}, h_{2}=k_{2}-\rho P_{11},
$$

for some entire function $\rho$. We now want to further modify $\left(h_{1}, h_{2}\right)=\vec{h}$ so to obtain a solution $\left(h_{1}^{*}, h_{2}^{*}\right)$ for the system (4.6), with $h_{i}^{*} \in \hat{\mathcal{D}}\left(K_{\varepsilon}\right)$. To achieve this result, it will be sufficient to look for $\lambda \in \hat{\mathcal{D}}\left(K_{\varepsilon}\right)$ such that

$$
h_{1}^{*}=h_{1}-\lambda P_{12}, h_{2}^{*}=h_{2}+\lambda P_{11}
$$

satisfy the following system:

$$
\left\{\begin{array}{l}
P_{11} h_{1}+P_{12} h_{2}=\hat{g}_{1} \\
P_{21}\left(h_{1}-\lambda P_{12}\right)+P_{22}\left(h_{2}+\lambda P_{11}\right)=\hat{g}_{2} \\
P_{13}\left(h_{1}-\lambda P_{12}\right)+P_{32}\left(h_{2}+\lambda P_{11}\right)=\hat{g}_{3}
\end{array}\right.
$$

The second and the third equations of (4.9) show that such a $\lambda$, if it exists, must satisfy the following system

$$
\left\{\begin{array}{l}
\lambda M_{12}=\hat{g}_{2}-h_{1} P_{21}-h_{2} P_{22} \\
\lambda M_{13}=\hat{g}_{3}-h_{1} P_{31}-h_{2} P_{32}
\end{array},\right.
$$

where

$$
\begin{aligned}
& M_{12}=P_{11} P_{22}-P_{21} P_{12}, \\
& M_{13}=P_{11} P_{32}-P_{31} P_{12} .
\end{aligned}
$$

We now note that if we set

$$
A_{1}=\left(\hat{g}_{2}-h_{1} P_{21}-h_{2} P_{22}\right) M_{13}
$$

and

$$
A_{2}=\left(\hat{g}_{3}-h_{1} P_{31}-h_{2} P_{32}\right) M_{12},
$$

a simple computation shows that

$$
\begin{aligned}
A_{1}-A_{2}= & k_{1}\left\{P_{21} M_{13}-P_{31} M_{12}\right\}+k_{2}\left\{P_{22} M_{13}-P_{32} M_{12}\right\} \\
& +h_{1}\left\{P_{31} M_{12}-P_{21} M_{13}\right\}+h_{2}\left\{P_{32} M_{12}-P_{22} M_{13}\right\} .
\end{aligned}
$$

We now observe (as in Theorem 3.2) that, for

$$
M_{23}=P_{22} P_{31}-P_{21} P_{32},
$$

one has

$$
P_{11} M_{23}=P_{21} M_{13}-P_{31} M_{12}
$$


and

$$
P_{12} M_{23}=P_{22} M_{13}-P_{32} M_{12} .
$$

By inserting (4.12) and (4.13) into (4.11) we get

$$
A_{1}-A_{2}=\left(k_{1}-h_{1}\right) P_{11} M_{23}+\left(k_{2}-h_{2}\right) P_{12} M_{23},
$$

and now, by (4.8),

$$
A_{1}-A_{2}=0 .
$$

This result shows us that the function

$$
\lambda=\frac{\hat{g}_{2}-h_{1} P_{21}-h_{2} P_{22}}{M_{12}}=\frac{\hat{g}_{3}-h_{1} P_{31}-h_{2} P_{32}}{M_{13}}
$$

is holomorphic in $\mathbb{C}^{n} \backslash\left\{z \in \mathbb{C}^{n}: M_{12}(z)=M_{13}(z)=0\right\}$. But, again by the regularity hypothesis, codim $\left\{z \in \mathbb{C}^{n}: M_{12}(z)=M_{13}(z)=0\right\}=2$, and therefore, by the Riemann extension theorem, we have $\lambda \in H\left(\mathbb{C}^{n}\right)$. Finally, by the Ehrenpreis-Malgrange Lemma (i.e. by Theorem 3.1), we deduce that $\lambda \in \hat{\mathcal{D}}\left(K_{\varepsilon}\right)$. Going back to (4.9), we finally have a solution $\vec{h}^{*}=\left(h_{1}^{*}, h_{2}^{*}\right), h_{i}^{*} \in$ $\hat{\mathcal{D}}\left(K_{\varepsilon}\right)$, to

$$
\sum_{j=1}^{3} P_{i j} h_{j}^{*}=\hat{g}_{i} .
$$

Now set $h_{j}^{\prime} \in \mathcal{D}\left(K_{e}\right)$ such that $\hat{h}_{j}^{\prime}=h_{j}^{*}$, and if we put $f_{j}^{*}=\tilde{f}_{j}-h_{j}^{\prime}$, the result is proved. The reader will notice that no new phenomenon takes place when $s, q$ grow, since one can repeat these arguments for every row of the system 4.6 , in an inductive way. More precisely, one can apply division to the first equation in (4.5) to obtain a solution $\vec{h}=\left(h_{1}, h_{2}, \ldots, h_{s}\right) \in \hat{\mathcal{D}}\left(K_{\varepsilon}\right)$; one then considers all possible solutions which differ from $\vec{h}$ by the elements of the first syzygy of $\left(P_{11}, \ldots, P_{1 \mathrm{~s}}\right)$; when replacing this into the second equation of $(4.5)$ one has to deal with the ideal generated by the $2 \times 2$ minors of the matrix formed by the first two rows of $P$; the division argument can, once again, be applied, and we now have a solution to the first two equations in (4.5) with elements in $\hat{\mathcal{D}}\left(K_{\varepsilon}\right)$. This procedure can be repeated until we get to the $s-t h$ equation. We now have $q-s+1$ equations in which an unknown function $\lambda$ multiplies $q-s+1$ minors of order $s$ of the matrix $P$ (the minors formed by the first $s-1$ rows and by another row among the remaining ones). One then concludes the right growth on $\lambda$, by employing the regularity of the matrix $P$.

Remark. The second part of the proof of Theorem 4.1 shows, indeed, a sort of faithful fiatness of $\hat{\mathcal{D}}(K)$ over $\mathbb{C}\left[z_{1}, \ldots, z_{n}\right]$, as long as some regularity conditions are imposed.

Remark. Theorem 4.1 shows the removability of singularity for $C^{\infty}$ solutions of rectangular systems of linear constant coefficients partial differential 
equations. There is, however, no change in the proof if one wishes to consider distribution solutions of the same system, or even ultradistributions (see [17], [18] for their definition and properties). For the readers convenience, we will just state the theorem for distribution solutions, and we leave it to the reader to provide the details of the proof.

Theorem 4.2. Let $P=\left[P_{i j}\right]$ be a $q \times s$ strongly regular matrix of polynomials in $\mathbb{C}^{n}$, for $1 \leq s \leq q, q \geq 2, n \geq 2$. Let $P_{i j}(D)$ denote the corresponding differential operators. Let $\Omega \subseteq \mathbb{R}^{n}$ be bounded and convex, and suppose $K \subseteq \Omega$ be compact and convex. Then, if $\vec{f}=\left(f_{1}, \ldots, f_{0}\right), f_{j} \in \mathcal{D}^{\prime}(\Omega \backslash K)$, satisfies, on $\Omega \backslash K$

$$
\sum_{j=1}^{s} P_{i j}(D) f_{j}=0, i=1, \ldots, q
$$

For any $\varepsilon>0$, one can find a unique element $\vec{f}^{*}=\left(f_{1}^{*}, \ldots, f_{a}^{*}\right), f_{i} \in \mathcal{D}^{\prime}(\Omega)$ such that

$$
\sum P_{i},(D) f_{j}^{*}=0, i=1, \ldots, q
$$

on all of $\Omega$, and $f_{j}^{*}$ coincides with $f_{j}$ on $\Omega \backslash K_{\epsilon}$ for $K_{\epsilon}$ en $\varepsilon$-neighborhood of $K$.

An unpleasant phenomenon which occurs in both these theorems is the fact that, when extending $\vec{f}$, it is not possible to obtain the extension up to $\partial K$, but in a little neighborhood of $\partial K$ we have that $\vec{f}$ and $\vec{f}^{*}$ may not coincide. This is of course a consequence of the non flabbiness of the sheaves $\mathcal{E}$ and $\mathcal{D}^{\prime}$, and can be removed if we get to work in the framework of the theory of the sheaf $\mathcal{B}$ of hyperfunctions. In this case we can even work with infinite order differential operators, instead of being restricted (as in the smooth case) to finite order operators. Let us introduce, here, the necessary notations and definitions, while referring the reader to [15], [26] for further details. Let $\mathcal{B}$ denote the sheaf of hyperfunctions on $\boldsymbol{R}^{n}$; its definition is fairly complicated but, for $\Omega$ an open set in $R^{n}$, one has

$$
\mathcal{B}(\Omega)=H_{\Omega}^{\mathrm{n}}(V, \mathcal{O})
$$

where $V$ is a complex neighborhood of $\Omega$ in $C^{n}, \mathcal{O}$ is the sheaf of germs of holomorphic functions and $H_{\Omega}^{\mathrm{n}}(V, \mathcal{O})$ denotes the relative cohomology group $H^{n}(V, V \backslash \Omega ; \mathcal{O})$. In many concrete instances, it is useful to consider the representation of hyperfunctions as equivalence classes of holomorphic functions and, eventually, as "boundary values" of holomorphic functions, but we shall not use this interpretation in this paper. What we will need, on the other hand, is the (highly nontrivial) fact that $\mathcal{B}$ is a flabby sheaf (i.e., the natural restriction map $B\left(\Omega_{1}\right) \rightarrow \mathcal{B}\left(\Omega_{2}\right), \Omega_{1} \supseteq \Omega_{2}$ open sets, is always surjective) and that if $K \subset \subset \mathbf{R}^{n}$ is a compact set, then the space

$$
\mathcal{B}_{K}\left(\mathbf{R}^{n}\right)
$$


of hyperfunctions supported in $K$ can be identified (by the Köthe duality theorem) with the space

$$
(\mathcal{O}(K))^{\prime}
$$

of analy tic functionals carried by $K$; this identification is particularly important because it allows us to define the space $\hat{\mathcal{B}}_{K}$ of Fourier-Borel transforms of the elements in $\mathcal{B}_{K}$, by simply applying the Fourier-Borel transform to $(\mathcal{O}(K))^{\prime}$. It is therefore possible to see that

$$
\begin{aligned}
\hat{\mathcal{B}}_{K} \cong\left\{F \in H\left(\mathbb{C}^{n}\right): \forall \varepsilon>03 A_{\varepsilon}>0\right. \text { s.t. } \\
|F(z)| \leq A_{\varepsilon} \exp \left(H_{K}(z)+\varepsilon|z|\right\},
\end{aligned}
$$

for $H_{K}(z)$ the supporting function of $K$. Finally two words on infinite order differential operators. When $K=\{0\}$, the space

$$
\begin{gathered}
\hat{\mathcal{B}}=(\mathcal{O}(\{0\}))^{\prime} \cong\left\{F \in H\left(\mathbb{C}^{n}\right): \forall \varepsilon>0 \exists A_{\varepsilon}>0\right. \text { s.t. } \\
\left.|F(z)| \leq A_{\varepsilon} \exp (\hat{\epsilon}|z|)\right\},
\end{gathered}
$$

is the space of entire functions of order one and minimal type (or of exponential type zero), which are also called, in Sato's own terminology, functions of infraexponential type. If $P(z) \in \hat{\mathcal{B}}_{\{0\}}$, one can consider the analytic functional $\mu \in(\mathcal{O}\{0\})^{\prime}$ such that $\hat{\mu}=P$ and consider the convolution operator

$$
\mu *: \mathcal{B} \longrightarrow \mathcal{B}
$$

Because $\mu$ is supported by the origin, the operator $\mu *$ is actually a local operator (also denoted by $P(D)$ ) which goes under the name of infinite order differential operator, and it acts as a sheaf homomorphism on the sheaf $\mathcal{B}$. With these notations we can finally prove the following result

Theorem 4.3. Let $R$ be the ring of entire functions of infraexponential type and let $P=\left[P_{i}\right]$ be a regular $q \times s$ matrix of entire functions of infraexponential type, in $R$. Suppose, moreover, that the $j \times j$ minors $(j=1, \ldots, s-1)$ which appear in Definition 4.1 form a slowly-decreasing $(s-j+1)$-tuple in $(\hat{O} \hat{(}\{0\}))^{\prime}$. Then if $\Omega \subseteq \mathbb{R}^{n}$ is a bounded open set, $K \subseteq \Omega$ is a compact convex set and $\vec{f}=\left(f_{1}, \ldots, f_{s}\right), f_{s} \in \mathcal{B}(\Omega \backslash K)$ satisfies, on $\Omega \backslash K$,

$$
\sum_{j=1}^{s} P_{i j}(D) f_{j}=0, i=1, \ldots, q
$$

one can find a unique element

$$
f^{*}=\left(f_{1}^{*}, \ldots, f_{s}^{*}\right), f_{j}^{*} \in \mathcal{B}(\Omega)
$$


such that

$$
\sum_{j=1}^{s} P_{i j}(D) f_{j}^{*}=0, i=1, \ldots, q,
$$

on all of $\Omega$, and $f_{j}^{*}$ coincides with $f_{j}$ on $\Omega \backslash K$.

Proof: The argument in the proof goes as for Theorem 4.1, with a few minor modifications. Indeed, by the fiabbiness of $\mathcal{B}$, we can find $\tilde{f}_{j} \in \mathcal{B}(\Omega)$ such that $\tilde{f}_{j}=f_{j}$ on $\Omega \backslash K$ (no loss on the boundary). Set

$$
g_{i}=\sum_{j=1}^{s} P_{i j}(D) \tilde{f}_{j}, t=1, \ldots, q ;
$$

one clearly has $g_{i} \in \mathcal{B}_{K}(\Omega)$, and

$$
\sum_{i=1}^{q} Q_{j t}(D) g_{t}=0
$$

for $Q=\left[Q_{j t}(D)\right]$ a matrix of infinite order differential operators contained in the module of relations of the rows of $P$. Fourier transform of (4.14), jointly with the hypothesis of regularity on $P$, shows the existence of entire functions $k_{1}, \ldots, k_{s}$ such that

$$
\hat{g}_{i}=\sum_{j=1}^{s} P_{i j} k_{j} \quad i=1, \ldots, q .
$$

One now has to modify the functions $k_{j}$ into elements in $\hat{\mathcal{B}}_{h}$, thus concluding the proof. This is done inductively on the equations in (4.15), by replacing Ehrenpreis' division theorem by Theorem 3.3. Indeed, assume we have solved the first $p$ equations of $(4.15), 1 \leq p \leq s-2$, with $\tilde{k}_{j} \in \hat{\mathcal{B}}_{K}$. We now modify $\left(\tilde{k}_{1}, \ldots, \tilde{k}_{s}\right)$ by adding elements in the module of relations of the first $p$ rows of $P$. These elements will be written by using the $(s-2) \times(s-2)$ minors of the matrix obtained by considering the first $s-2$ rows of $P$. When this is inserted in the $(p+1)-$ th equation of $(4.15)$ one obtains an equation such as

$$
\sum \lambda_{i} M_{i}=k
$$

for $k \in \hat{\mathcal{B}}_{K}$ given, $M_{i}$ the $(s-1) \times(s-1)$ minors of the matrix obtained with the first $(s-1)$ rows of $P$, and the $\lambda_{j}$ 's to be determined. Unlike what we did in Theorem 4.1, we cannot apply division directly to (4.16) since the $M_{i}$ will never by a slowly decreasing (or even a regular) sequence. But, as we already did twice in our previous results, (4.16) can be suitably modified (by multiplying by some $P_{i j}$, whose growth does not affect $\hat{\mathcal{B}}_{K}$ ) to leave a new equation

$$
\sum \mu_{j} N_{j}=k^{\prime}
$$


where $k^{\prime} \in \hat{\mathcal{B}}_{K}$, and the $N_{j}$ are exactly the minors which appear in the Definition 4.1 of strong regularity. Theorem 3.3 can now be applied to obtain the right growth on the $\mu_{j}$ 's, and the proof is then concluded as in Theorem 4.1. At the last step, finally, the first $(s-1)$ equations have been solved, and if one adds now their syzygies, the last $q-s+1$ equations of (4.16) provide a function which is holomorphic outside the set of common zeroes of the $s \times s$ minors of $P$, and is therefore entire. Finally its growth can be controlled, as every infraexponential type function is, in itself, slowly decreasing and the proof can now be completed as in Theorem 4.1.

Our last theorem finally deals with the case in which convolution operators in the spaces $\mathcal{E}$ and $\mathcal{D}^{\prime}$ replace partial differential operators. This result generalizes what the authors previously proved in [23].

In this case, we consider $\vec{f}=\left(f_{1}, \ldots, f_{s}\right)$, with $f_{j} \in \mathcal{E}(\Omega \backslash K)$, and $\mu=$ $\left[\mu_{i j}\right]$ a $q \times s$ matrix of compactly supported distributions such that $\mu_{j} \epsilon$ $\mathcal{E}^{\prime}\left(\mathbb{R}^{n}\right)$, ch supp $\left(\mu_{i j}\right)=T, T$ a compact convex set in $\mathbb{R}^{n}$, the same for all the distributions $\mu_{i j}$. Since we now have (section 3) a division theorem in the space $\hat{\mathcal{D}}(K)$, there is no difficulty in reproducing a theorem as before. Let us just show how to prove the necessary bounds, since the $j \times j$ minors of the matrix correspond, of course, to distributions supported in $j T$, and therefore some extra care may be needed. Let us therefore assume that, for $\mu_{i j}$ as above, and $\hat{g}_{i} \in \hat{\mathcal{D}}(K)$ we have entire functions $k_{1}, k_{2}$ such that

$$
\left\{\begin{array}{l}
\hat{\mu}_{11} k_{1}+\hat{\mu}_{12} k_{2}=\hat{g}_{1} \\
\hat{\mu}_{21} k_{1}+\hat{\mu}_{22} k_{2}=\hat{g}_{2} \\
\hat{\mu}_{31} k_{1}+\hat{\mu}_{32} k_{2}=\hat{g}_{3}
\end{array}\right.
$$

We must show that the $k_{j}$ 's can be modified so that their growth provides elements in $\hat{\mathcal{D}}\left(K_{\varepsilon}-T\right)$. We argue as in Theorems $3.3,3.3$; if we assume $\left(\hat{\mu}_{11}, \hat{\mu}_{12}\right)$ to be $T$-slowly decreasing we have that $h_{1}, h_{2}$ can be found in $\hat{\mathcal{D}}\left(K_{e}-\right.$ $T)$, so that

$$
\hat{\mu}_{11} h_{1}+\hat{\mu}_{12} h_{2}=\hat{g}_{1}
$$

and

$$
h_{1}=k_{1}+\rho \hat{\mu}_{12}, h_{2}=k_{2}-\rho \hat{\mu}_{11},
$$

for some entire function $\rho$.

We now seek $\lambda \in \hat{\mathcal{D}}\left(K_{\varepsilon}-2 T\right)$ such that

$$
h_{1}^{*}=h_{1}-\lambda \hat{\mu}_{12}, h_{2}^{*}=h_{2}+\lambda \hat{\mu}_{11}
$$

satisfy the system (4.18) (the reason for the $K_{\varepsilon}-2 T$ is that we seek $h_{i}^{*} \in$ $\left.\hat{\mathcal{D}}\left(K_{\epsilon}-T\right)\right)$. But arguing as in the proof of Theorem 4.I, we see that $\lambda$ is given by the entire function (if regularity is assumed on the matrix $\hat{\mu}$ )

$$
\frac{\hat{g}_{2}-h_{1} \hat{\mu}_{21}-h_{2} \hat{\mu}_{22}}{M_{12}}=\frac{\hat{g}_{3}-h_{1} \hat{\mu}_{31}-h_{2} \hat{\mu}_{32}}{M_{13}} ;
$$

if now we know that $M_{12}$ is $2 T$-slowly decreasing, we deduce the right growth on $\lambda$. This shows than we can now state the following resuits: 
Theorem 4.4. Let $\mu=\left[\mu_{i j}\right]$ be a $q \times s$ matrix $(q \geq s)$ of compactly supported distributions, and suppose ch $\operatorname{supp}\left(\mu_{i j}\right)=T$ for any $i, j$. Assume furthermore, that the matrix $\hat{\mu}=\left[\hat{\mu}_{i j}\right]$ is regular in $H\left(\mathbb{C}^{n}\right)$, that the $j \times j$ minors $(j=$ $i, \ldots, s-1)$ of Definition 4.1 form a $j T$-slowly decreasing $(s-j+1)$-tuple in $\hat{\mathcal{E}}^{\prime}$ and finally, that each $s \times s$ minor in $\hat{\mu}$ is, as well, sT-slowly decreasing. Then if $\Omega \subseteq \mathbb{R}^{n}$ is a bounded open set, $K \subseteq \Omega$ is a compact convex set and $\vec{f}=\left(f_{1}, \ldots, f_{s}\right) \in \mathcal{E}(\Omega \backslash K)$ satisfies, on $(\Omega \backslash K)-T$,

$$
\sum_{j=1}^{s} \mu_{i j} * f_{j}=0, i=1, \ldots, q
$$

and $\varepsilon>0$, one can find a unique element $\vec{f}^{*}=\left(f_{1}^{*}, \ldots, f_{s}^{*}\right), f_{j}^{*} \in \mathcal{E}(\Omega)$ such that, on $(\Omega \backslash K)-T$,

$$
\sum_{j=1}^{s} \mu_{j} * f_{j}^{*}=0, i=1, \ldots, q
$$

and $f_{j}^{*}$ coincides with $f_{j}$ on $\Omega \backslash K_{\varepsilon}$, for $K_{\varepsilon}$ an $\varepsilon$-neighborhood of $K$.

Theorem 4.5. The same result holds for distribution solutions.

The reader can finally provide examples to Theorems $4.3,4.4,4.5$ by considering infinite products as indicated in [15], as far as Theorem 4.5 is concerned, or exponential sums and exponential polynomials for Theorems 4.4 and 4.5 . We refer the reader to [3] and [4] for these constructions.

\section{References}

[1] A. ANdreotti and M. Nacinovich, Complexes of partial differential operators, Ann. Scuola Norm. Sup. Pisa 3 (1976), 553-621.

[2] D. BAYER AND M. STILlMAN, On the complexity of computing syzygies, J. Symbolic Comp. (to appear).

[3] C.A. BERENSTEIN AND D.C. STRuppa, Solutions of convolution equations in convex sets, Amer. J. Math. 109 (1987), 521-544.

[4] C.A. BERENSTEIN AND B.A. TAYLOR, Interpolation problems in $C^{n}$ with applications to harmonic analysis, J. Anal. Math. 38 (1980), 188-254.

[5] S. Bochner, Partial differential equations and analytic contintation, Proc. Nat. Ac. Sc. 38 (1952), 227-230.

[6] D. Buchsbaum, A generalized Koszul complex I, Trans. Amer. Math. Soc. 111 (1964), 183-196. 
[7] D. Buchsbaum AND D. RIM, A generalized Koszul complex II, depth and multiplicity, Trans. Amer. Math. Soc. 111 (1964), 197-224.

[8] J.A. EAGON AND D.G. NorThCoTt, Ideals defined by matrices and a certain complex associated with them, Proc. Roy. Soc. of London 269 (1962), 188-204.

[9] L. EHRENPREIS, Some applications of the theory of distributions to several complex variables, Seminar on analytic functions 1, Princeton (1957), 66-79.

[10] L. EHRENPREIS, A new proof and an extension of Hartogs' theorem, Bull. Am. Math. Soc. 67 (1961), 507-509.

[11] L. EHREn Preis, "Fourier Analysis in Several Complex Variables," Wiley Interscience, New York, 1970.

[12] G. HERMANN, Die frage der entlich rielen Schritte in der Theorie der Polynomideale, Math. Ann. 9 (1926), 736-788.

[13] D. HILBERT, Uber die vollen Invariantensysteme, Math. Ann. 42 (1893), 313-373.

[14] A. KANEKO, On Hartogs type continuation theorem for regular solutions of linear partial differential equations with constant coeficients, $J$. Fac. Sci. Univ. Tokyo Sect. IA 35 (1988), 1-26.

[15] T. KaWAI AND D.C. STRuppa, An existence theorem for holomorphic solutions of infinite order linear differential cquations with constant coefficients, Int. J. Math. 1 (1990), 63-82.

[16] J.J. KELLEHER AND B.A. TAYLOR, On finitely generated modules over some rings of analytic functions, manuscript.

[17] H. Komatsu, Ultradistributions I, Structure theorems and a characterization, J. Fac. Sci. Univ. Tokyo 20 (1973), 25-105.

[18] H. Komatsu, Ultradistributions II, The Kernel theorem and ultradistributions with support in a manifold, J. Fac. Sci. Uni. Tokyo 24 (1977), $607-628$.

[19] D. LAZARD, Algèbre linéaire sur $K\left[X_{1}, \ldots, X_{n}\right]$ et élimination, Bull. Soc. Math. France 105 (1977), 165-190.

[20] J. Leray, Séminaire sur les équations aux dérivées partielles, Collège de France, 1961-1962.

[21] F.S. MaCaulay, "The Algebraic Theory of Modular Systems," Cambridge Tracts 19, 1916.

[22] H. Matsumura, "Commutative Algebra," Benjamin Cummings Publ. Co, 1980.

[23] A. Meril and D.C. Struppa, "Phénomène de Hartogs et équations de convolution," Springer Lecture Notes in Math 1295, 1987, pp. 146-156.

[24] D.G. NorthcotT, "Lesson on Rings, Modules and Multiplicities," Cambridge U.P., 1968. 
[25] V.P. Palamodov, "Linear Differential Operators with Constant Coefficients," Springer Verlag, Berlin, 1970.

[26] M. Sato, T. Kawai and M. Kashiwara, "Microfunctions and pseudo differential equations," Proc. of a conference at Kataka, Lecture Notes in Math. Springer 187, 1973, pp. 265-529.

[27] D.C. STRuPpA, The first eighty years of Hartogs' theorem, Sen. di Geometria Univ. Bologna, 1987-88, 127-211.

[28] O. Zariski and P. SamUel, "Commutative Algebra," Van Nostrand, Princeton, 1958.

[29] C.A. Berenstein AND M. DOSTAL, "Analytically uniform spaces and their applications to convolution equations," Springer Lecture Notes in Mathematics 256, 1972.

[30] D.C. STRUPPA, The fundamental principle for systems of convolution equations, Mem. A.M.S. 273 (1983).

A. Meril: Department of Mathematics

University of Antilles

F.W.I.

D.C. Struppa: Department of Mathematical Sciences

George Mason University

Fairfax, Virginia 22030

U.S.A.

Rebut el 12 de Gener de 1990 